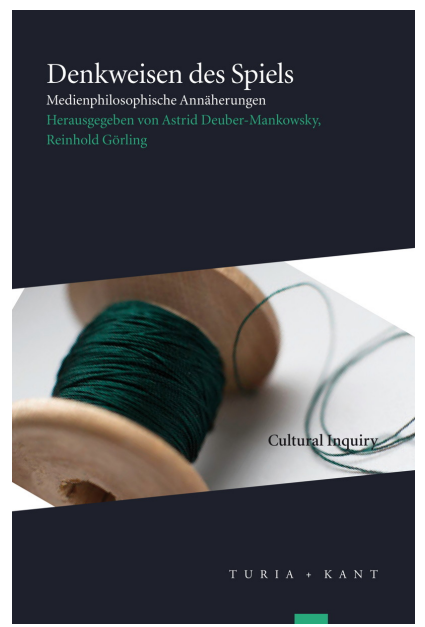

Denkweisen des Spiels: Medienphilosophische Annäherungen, hg. v. Astrid Deuber-Mankowsky und Reinhold Görling, Cultural Inquiry, 10 (Wien: Turia + Kant, 2017), S. 155-70

\section{SERJOSCHA WIEMER}

\section{Niemandes Spiel?}

Zur Aufteilung des Spielbegriffs oder: Die Schwierigkeit, die spielende Maschine zu denken

\author{
ZITIERVORGABE:
}

Serjoscha Wiemer, »Niemandes Spiel?: Zur Aufteilung des Spielbegriffs oder: Die Schwierigkeit, die spielende Maschine zu denken $\ll$, in Denkweisen des Spiels: Medienphilosophische Annäherungen, hg. v. Astrid Deuber-Mankowsky und Reinhold Görling, Cultural Inquiry, 10 (Wien: Turia + Kant, 2017), S. 155-70 <https://doi.org/10.37050/ ci-10_09>

\section{ANGABE ZU DEN RECHTEN:}

(C) by the author(s)

This version is licensed under a Creative Commons AttributionShareAlike 4.0 International License. 


\section{NIEMANDES SPIEL?}

Zur Aufteilung des Spielbegriffs oder: Die Schwierigkeit, die spielende Maschine zu denken

Serjoscha Wiemer

Das Spiel wird im 20. Jahrhundert zu einem festen Bestandteil von Wissensproduktion, beeinflusst Theoriebildung, Experiment und Simulation. In unterschiedlichen Kontexten und wissenschaftlichen Disziplinen, von der Philosophie über die Mathematik bis zur Biologie, von der Ökonomie bis zur Softwaretheorie werden Spiele und Spielbegriffe verwendet. Die vielfältige Produktivität des Spiels gründet jedoch nicht in einer übergreifenden und einheitlichen Auffassung. Im Gegenteil: Die Verschiedenheit der Spielbegriffe ist unübersehbar. Allerdings lässt sich behaupten, dass eine spezifische Attraktivität von Spielen offenbar darin besteht, mit ihrer Hilfe dynamische Vorgänge darstellen, beschreiben und modellieren zu können. Dies gilt insbesondere dann, wenn Prozess und Verzeitlichung, Aktualisierung oder Aufführung oder allgemein der spielerische Vollzug als Qualitäten oder vielleicht sogar als notwendige Entfaltung von `Spiek gedacht werden. Im Vollzug des Spiels als 'Spielen ereignet sich allerdings mehr als die Aktualisierung der Struktur eines Regelwerks oder einer Vor-Schrift. Die Bewegung als Prinzip einer zeitlichen Differenzproduktion steht in Spannung zur begrifflichen Feststellung im Sinne abstrakter oder der Zeit enthobener Identität. Das Spiel ist im Spielen nicht mit sich gleich, sondern verschieden. ${ }^{1}$ Spiel steht darum in unterschiedlichen Diskursen für die Produktion von Differenz. ${ }^{2}$

1 Eine ähnliche Unterscheidung zwischen Form und Vollzug als Problem der Differenz zwischen Fixierung und Bewegung ist in der systemtheoretischen Diskussion behandelt worden. Vgl. Sybille Krämer, »Form als Vollzug oder: Was gewinnen wir mit Niklas Luhmanns Unterscheidung von Medium und Form? «, Rechtshistorisches Journal, 17 (1998), S. 558-73.

2 Der Zusammenhang von Spiel und Differenz ist prägnant von Heidegger in Identität und Differenz behauptet worden. Vgl. Martin Heidegger, Identität und Differenz (Pfullingen: Neske, 1986), S. 56-58. Einflussreich für die Verbindung von Spiel und Differenzproduktion in philosophisch-medientheoretischer Perspektive 


\section{AUFSPALTUNG DES SPIELBEGRIFFS}

Trotz der anregenden Vielfalt unterschiedlicher Spielbegriffe ist eine übergreifende Polarisierung oder Aufspaltung im Nachdenken über das Spiel beobachtbar. So als wäre der Spielbegriff in sich selbst zweigeteilt. Beide Pole können zugespitzt gegenübergestellt werden: Auf der einen Seite herrscht ein Spielverständnis, das man 'kulturalistisch könnte. Aus dieser Perspektive wird das Spiel häufig als kulturelle oder kulturstiftende Institution betont. Alles Spielen muss nach dieser Auffassung durch ein ihm zugehöriges Spielbewusstsein oder eine charakteristische Haltung begleitet sein. Spiel, so die zugehörige Idee, ist nicht zu trennen von einem Spielbewusstsein, von einem die Handlung begleitenden Wissen um den der übrigen Welt enthobenen Charakter des Spiels. Die spieltheoretischen Studien von Johan Huizinga (1938) und Roger Caillois (1958) werden immer wieder als Referenzen für einen solchen Spielbegriff herangezogen. Bei Huizinga heißt es in Homo Ludens. Vom Ursprung der Kultur im Spiel:

Spiel ist eine freiwillige Handlung oder Beschäftigung, die innerhalb gewisser festgesetzter Grenzen von Zeit und Raum nach freiwillig angenommenen, aber unbedingt bindenden Regeln verrichtet wird, ihr Ziel in sich selber hat und begleitet wird von einem Gefühl der Spannung und Freude und einem Bewußtsein des >Andersseins` als das >gewöhnliche Leben ${ }^{3}$

In Roger Caillois' Studie Die Spiele und die Menschen. Maske und Rausch findet man eine ebenfalls kanonische Definition des Spiels, die einem >kulturalistischen Spielverständnis als Belegstelle dient: ${ }^{4}$

ist Derridas Konzept der Différance. Vgl. Jacques Derrida, »Die différance«, in ders., Randgänge der Philosophie (Wien: Passagen-Verlag, 1988), S. 31-52.

Johan Huizinga, Homo Ludens. Vom Ursprung der Kultur im Spiel (Reinbek bei Hamburg: Rowohlt, 2006), S. 37.

4 Caillois' Spielbegriff wäre eine eigene Untersuchung wert und das Zitat steht hier nicht stellvertretend für seine Theorie insgesamt, sondern lediglich für eine bestimmte Lesart. Caillois fokussiert in seiner Studie zwar das Spiel als eine Instanz menschlicher Kultur, doch mit seinem Spieltrieb-Konzept nimmt er auf einen viel weiter gefassten Spielbegriff Bezug, der auch das Verhalten von Tieren und die organischen Verwandlungen von Insekten mit einschließt. Die Mimikry von Insekten, bezeichnet Caillois als »Äquivalent für die Verwandlungsspiele des Menschen", vgl. Roger Caillois, Die Spiele und die Menschen. Maske und Rausch (Frankfurt a.M., Berlin, Wien: Ullstein, 1982), S. 28-29. Bemerkenswert 
Das Spiel ist [...] eine fiktive Betätigung, die von einem spezifischen Bewußtsein einer zweiten Wirklichkeit oder einer in Bezug auf das gewöhnliche Leben freien Unwirklichkeit begleitet wird. ${ }^{5}$

Die Betonung von Freiwilligkeit und "Bewusstsein « - ein Bewusstsein, welches das Spiel als Spiel reflektiert und die Tätigkeit des Spielens begleiten soll - ist kennzeichnend für den anthropozentrischen Zug dieser Spielkonzeption. Auf der anderen Seite steht ein Spielverständnis, das man als >formalistisch charakterisieren könnte. Es tritt prägnant in der mathematischen Behandlung von Spielen hervor, findet sich jedoch in vielen unterschiedlichen Disziplinen wieder, die mathematische Verfahren nutzen. Aus dieser Sichtweise ist es möglich, Spiele als mathematische Regelgebilde, als Systeme, Algorithmen oder Automaten zu konzipieren, deren Eigenschaften rein formal bestimmt werden können. Das >formalistische< Spielverständnis kommt darum ohne zentrale Bezugnahme auf den >Menschen aus, ohne Bewusstsein und ohne die subjektive Qualität einer spezifisch spielerischen `Haltung ${ }^{6}{ }^{6}$

ist die Richtung der Analogie, die weder logisch noch naturtheoretisch überzeugend ist. Man darf wohl davon ausgehen, dass die Körperverwandlungen der Insekten naturgeschichtlich das ältere Phänomen darstellen. Deswegen wäre es nur folgerichtig, die Verwandlungsspiele des Menschen nach dem Modell der Insektenmimikry zu denken, und nicht umgekehrt. Tatsächlich zeigt sich darin eine anthropozentrische Zuspitzung in Caillois' Argumentation. Natur, so könnte man diese Haltung verallgemeinernd charakterisieren, wird nach dem Modell von Kultur verstanden. In früheren Arbeiten hat Caillois die gegenteilige Argumentation erprobt und psychologisch charakterisierbare Wahrnehmungsstörungen (Schizophrenie) in Analogie zur organischen Insektenmimikry zu verstehen versucht. In Die Spiele und die Menschen distanziert er sich von diesem früheren Ansatz aus Mimese und legendäre Psychasthenie (1935), in Roger Caillois, Méduse \& Cie (Berlin: Brinkmann \& Bose, 2007), S. 25-43.

5 Caillois, Die Spiele, S. 16.

6 Prominent und historisch einflussreich für diesen formalistischen Zugang ist die mathematische Spieltheorie von John von Neumann und Oskar Morgenstern (1944), wenngleich in der dort perspektivierten Verwendung der Spieltheorie für ökonomische Analysen der Mensch als abstrakte Instanz von (utilitaristisch-strategischen) Entscheidungshandlungen im Horizont der Spiele verbleibt. Die mathematisch-ökonomische Spieltheorie behält den eigentümlichen Doppelcharakter, sowohl formales System als auch Theorie sozialer Tauschbeziehungen zu sein. Vgl. John von Neumann und Oskar Morgenstern, Theory of Games and Economic Behavior, hg. v. Ariel Rubinstein und Harold W. Kuhn (Princeton, NJ: Princeton University Press, 2007). 


\section{POLARISIERUNGEN VON SPIEL IN DER PHILOSOPHIEGESCHICHTE}

Die bis hierhin skizzierte Aufteilung des Spielbegriffs könnte so aufgefasst werden, als ob der Spielbegriff eine wissenschaftsgeschichtliche Situation der Trennung von kulturwissenschaftlichen und naturwissenschaftlichen Denkweisen widerspiegelt. Die gegenläufigen Pole des Spielverständnisses könnten als Ausdruck der unterschiedlichen Spezialisierungen der wissenschaftlichen Disziplinen und Kulturen verstanden werden. Allerdings wird auch erkennbar, dass seit dem Ende des 19. Jahrhunderts quer zu einzelnen Disziplinen insgesamt ein zunehmendes Interesse am Spiel sich entwickelt. Im Nachdenken über das Spiel werden dabei einerseits ältere Traditionen und Theorien fortgeschrieben, andererseits wird das Spiel aber auch im Ansatz >neu< zu denken versucht, und tritt in die Position eines Schlüsselbegriffs, um Fragen nach der Entwicklung, nach der Geschichte und dem `Funktionieren $<$ von Kultur, Technik und Natur zu stellen. In der Philosophiegeschichte stößt man allerdings auf eine der heutigen Situation zumindest in ihrer Polarisierung vergleichbare Zweiteilung im Nachdenken über das Spiel. Während in der einen Denktradition Spiel als menschliche Betätigung betont wird, steht demgegenüber ein Begriff von Spiel als Naturphänomen, als Spiel der Welt oder der >Götter .

Die Traditionslinie, in der Spiel als Spiel des Menschen betont wird, reicht mindestens zurück bis zu Platons Philosophie. Platon charakterisierte das Spiel als menschliche Tätigkeit und ordnete es als Mimesis den Künsten zu. Er setzte einen definierten Abstand zwischen Spiel und wirklicher Erkenntnis und wertete es damit ab. ${ }^{7}$ Bei Kant, Schiller oder Schleiermacher wird ebenfalls, allerdings unter umgekehrten Vorzeichen, das Spiel als dem Menschen eigene Tätigkeit gedacht und in einen anthropologischen Kontext gestellt. ${ }^{8}$ Eine Denkweise, die im 20. Jahrhundert von Huizinga und Caillois fortgeführt wird.

$\mathrm{Zu}$ den bemerkenswerten philosophischen Spielkonzeptionen, in denen die Welt als Spiel gedacht wird, zählt Heraklits berühmtes Frag-

7 A. Corbineau-Hoffmann, "Spiel«, in Historisches Wörterbuch der Philosophie, hg. v. Joachim Ritter und Karlfried Gründer (Basel: Schwabe \& Co., 1998), IX (1998), S. 1383-90 (S. 1384). »Auch die Künste verdienen nur die Bezeichnung Spiel, da sie, vom Menschen gemacht, Nachbilder und keine Urbilder sind «, fasst Corbineau-Hoffmann Platons Position zusammen.

8 Ebd., S. 1384.

I 58 
ment 52, in dem die Zeit (Äon/Aion) als ein sspielendes Kind beschrieben ist. Romantiker wie Schlegel oder Novalis kritisierten eine auf das menschliche Subjekt bezogene Bedeutung von Spiel als einengend und wollten künstlerische Produktion in Analogie zum »Welt-Spiel «, als dem »unendlichen Spiele der Welt «, verstehen. ${ }^{9}$ Nietzsches Spielkonzeption wurde ebenso dieser Tradition zugerechnet wie die historisch jüngeren philosophischen Spielbegriffe von Eugen Fink und Hans-Georg Gadamer.

\section{GADAMERS MÜCKEN - SPIEL ALS BEWEGUNGSFORM DER NATUR}

Hans-Georg Gadamers philosophischer Spielbegriff lässt sich in der Tradition einer Spiel-der-Welt-Ontologie verstehen, in der das Spiel unabhängig vom Menschen zu denken ist. Gadamer thematisiert das Spiel in Wabrheit und Methode im Rahmen seiner Ontologie des Kunstwerks, und zwar solcherart, dass die »Frage nach dem Wesen des Spiels selbst « in Absetzung zu der subjektiven Reflexion des Spielenden gestellt wird. ${ }^{10}$ Gadamer betont das Spiel in seiner Autonomie gegenüber dem Spielenden; stattdessen stellt er einen Begriff von Spiel als »Bewegungsform der Natur « ins Zentrum: ${ }^{11}$

Betrachten wir den Wortgebrauch von Spiel, indem wir die sogenannten übertragenen Bedeutungen bevorzugen, so ergibt sich: Wir reden vom Spiel des Lichtes, vom Spiel der Wellen, vom Spiel des Maschinenteils in einem Kugellager, vom Zusammenspiel der Glieder, vom Spiel der Kräfte, vom Spiel der Mücken, ja sogar vom Wortspiel. Immer ist da das Hin und Her einer Bewegung gemeint, die an keinem Ziele festgemacht ist, an dem sie endet. Dem entspricht die ursprüngliche Bedeutung des Wortes Spiel als Tanz $[\ldots]^{12}$

Ebd.

10 Hans-Georg Gadamer, Wahrheit und Methode. Grundzüge einer philosophischen Hermeneutik, 2. durch e. Nachtr. erw. Aufl. (Tübingen: Mohr (Siebeck), 1965 [1960]), S. 98.

11 Ebd., S. 100.

12 Ebd., S. 99. Gadamer bezieht sich für diese Lesart von Mimesis auf die Untersuchung von Hermann Koller, Die Mimesis in der Antike, Dissertationes Bernenses: Serie 1, Fasc. 5 (Bernae, Bern: Francke, 1954). Koller betont dort den ursprünglichen Zusammenhang von Mimesis und Tanz. 
An den Beispielen, die den Spielbegriff hier kennzeichnen, fällt auf, dass Gadamer in seiner Betonung der Bewegungsformen sehr heterogene Phänomenbereiche nennt. Physikalische, optische, geologische, maschinelle, organische, animalische ebenso wie sprachlich-semantische Gegebenheiten werden als Spiel bezeichnet. Nur innerhalb dieser heterogenen Vielheit wird auch dem spielenden Menschen eine Position zugewiesen. Ein solcher Spielbegriff relativiert die Stellung des menschlichen Spiels und richtet sich damit zugleich gegen eine subjektzentrierte, bewusstseinsphilosophische oder anthropozentrische Begründung des Spiels:

Daß die Seinsweise des Spiels derart der Bewegungsform der Natur nahesteht, erlaubt aber eine wichtige methodische Folgerung. Es ist offenbar nicht so, daß auch Tiere spielen und daß man im übertragenen Sinne sogar vom Wasser und vom Licht sagen kann, daß es spielt. Vielmehr können wir umgekehrt vom Menschen sagen, daß auch er spielt. Auch sein Spielen ist ein Naturvorgang. ${ }^{13}$

Bemerkenswert für die hier verfolgte Frage nach der Aufteilung des Spielbegriffs und der Polarität gegenläufiger Denkweisen ist vor allem, wie deutlich Gadamers Frage nach dem Wesen des Spiels und sein Entwurf einer Spiel-Ontologie geradezu als Umkehrung der anthropologischen Spielbegriffe aufgefasst werden können. ${ }^{14}$ Dazu gehört die klare Zurückweisung eines Spielbegriffs, demzufolge Spiel immer schon als die Handlung eines tätigen Subjekts und als Ausdruck freier Subjektivität aufzufassen wäre. So betont Gadamer, dass »alles Spielen [...] ein Gespielt-werden« sei:

13 Gadamer, Wahrheit, S. 100.

14 Für Gadamer ist der Spielbegriff in Wabrheit und Methode ein Sprungbrett für eine theoretische Bestimmung des Begriffs des Kunstwerks und seiner Autonomie. Auffallend ist, dass die Differenz zwischen menschlichem Spiel und dem Spiel der Natur bei Gadamer merkwürdig in der Schwebe bleibt. Das menschliche Spiel wird einerseits dem »Naturvorgang « des Spiels eingegliedert, andererseits aber wird der Spielcharakter der Kunst nicht als Verdoppelung eines Naturvorgangs aufgefasst, sondern als »Vollendung « des menschlichen Spiels. Gadamers Begriff der »Darstellung « kann als entscheidender Schnittpunkt zwischen Spiel und Kunstwerk angesehen werden. Spiel wird von Gadamer als ausgezeichnetes Beispiel für Selbstdarstellung als universaler »Seinsaspekt der Natur « (ebd.) verstanden, und analog wird der Aspekt der Darstellung als entscheidend für die Seinsweise des Kunstwerks gefasst (ebd., S. 111). 
Der Reiz des Spiels, die Faszination, die es ausübt, besteht eben darin, daß das Spiel über den Spielenden Herr wird. [...] Das eigentliche Subjekt des Spiels (das machen gerade solche Erfahrungen evident, in denen es nur einen einzelnen Spielenden gibt) ist nicht der Spieler, sondern das Spiel selbst. ${ }^{15}$

\section{FINK：SPIEL ALS WELTSYMBOL}

1960, im gleichen Jahr, in dem Gadamer Wabrheit und Methode veröffentlicht, erscheint auch Eugen Finks Spiel als Weltsymbol. ${ }^{16}$ Fink unterscheidet zwischen einem Denken des Spiels als "anthropologischem « und »kosmologischem "Schlüssel. Spiel basiere als ein »Schlüsselphänomen $"{ }^{17}$ auf einer simmanenten Wiederholung` oder »Spiegelung « des »Ganzen in einem ausgezeichneten Teil «. ${ }^{18}$ Verstanden als anthropologischer Schlüssel wird dem Spiel eine ausgezeichnete Rolle zugemessen, Aussagen über den Menschen und sein Mensch-Sein zu vermitteln, und zwar derart, dass »das Spiel das Wesen des Menschen sein soll «. ${ }^{19}$ Im Unterschied dazu ist für einen Begriff von Spiel als `kosmologischer Schlüssel< das Spiel der Welt, nicht der Mensch, entscheidend. Kosmologischer Schlüssel meint: Das Spiel begründet einen Zugang zum Verständnis der Welt und zwar derart, »daß das Spiel das Wesen des Seienden im Ganzen sein soll «. ${ }^{20}$

15

Eugen Fink, Spiel als Weltsymbol, Gesamtausgabe (Freiburg: Alber, 2006), VII. Für den Hinweis auf den Spielbegriff Finks bin ich Astrid Deuber-Mankowsky zu Dank verpflichtet, deren Bezugnahme auf Fink einigen der hier vorgestellten Überlegungen die Richtung gewiesen hat. Zu Finks Spielbegriff sowie zum Verhältnis von Spiel und Technik vgl. weiterführend Astrid Deuber-Mankowsky, "Spiel und zweite Technik. Walter Benjamins Entwurf einer Medienthropologie des Spiels «, in Mediale Anthropologie, hg. v. Christiane Voss u. Lorenz Engell (Paderborn: Fink, 2015), S. 35-62.

17 Fink verwendet den Begriff "Schlüsselphänomen « sehr bewusst. Er reflektiert dessen Gebrauch als Mittel der zielgerichteten Übertreibung: »Die Ansetzung eines `Schlüsselphänomens besagt eine spekulative Umwandlung des naiven Begriffs: gewisse Züge werden festgehalten, andere übertrieben u. ausgeweitet. « (Fink, Spiel, S. 268). Ebd., S. 270. Dies hält Fink 1954 in Notizen fest, die seinem Seminar zum philosophisch-pädagogischen Problem des Spiels zugehören. Ebd., S. 262-81.

19 Ebd., S. 268.

20 Ebd. 
In seiner Untersuchung zur Philosophie des Spiels versucht Fink die Herkunft beider Ansätze in einer Geschichte der abendländischen Philosophie und Metaphysik aufzuzeigen. ${ }^{21}$ Nach Fink gewinnt die abendländische Philosophie bei Platon in einem strategischen move ihr Selbstverständnis gerade aus der Abwertung des Spiels und in Auseinandersetzung mit diesem. Im Zuge seiner Dichterkritik weise Platon dem Spiel, gleichauf mit dem homerischen Epos und der attischen Tragödie, eine Stellung auf einer unteren Stufe der Wahrheit zu. Durch den »Einblick in die mimetische Natur des Spiels « könne er den Wettkampf mit Homer und den Tragikern gewinnen. ${ }^{22}$ Dadurch erst, so Fink, dass sie den Wirklichkeits- und Wahrheitsgehalt des Spiels herabsetzt, bekräftigt die platonische Metaphysik ihren eigenen Wahrheitsanspruch. ${ }^{23}$

Finks Kritik an der metaphysischen Abwertung des Spiels richtet sich insbesondere darauf, dass damit das Spiel als Nachahmung und abgeleitetes Phänomen nach dem Modell von Abbild und Urbild gedacht wird. Zwar gebe es eine strukturelle Ähnlichkeit zwischen "Bildwelt « und »Spielwelt «, ${ }^{24}$ doch bestehe der wesentliche Unterschied in der hervorbringenden Eigenschaft des Spiels. Nicht in seinem Verhältnis zu einem »Urbild « (und damit als Rückverweisung auf ein Original, demgegenüber es durch Abkünftigkeit und Nichtorginalität gekennzeichnet wäre), sieht Fink die definierende Qualität von Spiel, sondern in seiner Originalität und Produktivität. ${ }^{25}$ Dabei hebt er die Eigenart der spielerischen Produktion hervor, nicht als Produktion von etwas, sondern als spielerische Bewegung sich zu entfalten, als Spielen. »Die Spielproduktion kommt nicht in einem Resultat an. Oder anderes gewendet und in schärferer Antithese formuliert: Spielen ist als Produzieren von spielweltlichem Schein. ${ }^{26}$

Für Fink gewinnt das Spiel als ’kosmologischer Schlüssel in der Distanz zum platonischen >Schein< Kontur: »Das Weltall spielt: aber

21 Fink war einer der bedeutendsten Schüler Edmund Husserls und promovierte bei Husserl und Martin Heidegger. Finks Zugang zur Geschichte der Philosophie ist durch Heidegger mit geprägt. Vgl. zum Einfluss Heideggers auf Finks Begriffe von `Spiek und 'Welt « weiterführend Stuart Elden, »Eugen Fink and the question of the world «, Parrhesia: a journal of critical philosophy, 5 (2008), S. 48-59.

22 Fink, Spiel, S. 108.

23 Ebd.

24 Ebd., S. 114.

25 Ebd., S. 119.

26 Ebd., S. 116. 
weder als Person, noch spielt es so, daß es dabei einen `Schein`, eine ১Unwirklichkeit‘, eine imaginäre Szene erspielt. Wenn wir von einem Spiel der Welt reden wollen ", so Fink, »müssen wir Spielstrukturen des Menschenspiels entscheidend umdenken, und zwar in jenen Zügen umdenken, wodurch es als abgeleitet vom Walten der Welt her sich gibt ${ }^{27}$ Für einen solchen Spielbegriff ist das Spiel weder über seinen sunwirklichen`Status noch durch eine sals-ob< Haltung charakterisiert. Auch wird die notwendige Bindung an den Menschen oder eine spielerische Subjektivität ausgespart. Kann ein solcher Spielbegriff anders als durch Analogiebildungen und Metaphern verstanden werden? Das Spiel der Mücken, der Wellen, etc.? In Finks Spielphilosophie wird das Spiel selbst zum »kosmischen Gleichnis «, wenn das Spiel obne Spieler gedacht wird. ${ }^{28}$ Das Spiel ohne Personen, als >Niemandes Spiel<:

Die Spielmetapher versagt als kosmisches Gleichnis, wenn wir an der Personalität eines Spielers und an der Scheinhaftigkeit der spielweltlichen Szene festhalten. Nur in einer entscheidend gestörten und dadurch zerbrochenen >Gleichung können wir von einem Spiel der Welt reden. Das Spiel der Welt ist niemandes Spiel, weil es erst darin Jemande, Personen, Menschen und Götter gibt; und die Spielwelt des Weltspiels ist nicht ein 'Schein<, sondern die Erscheinung. ${ }^{29}$

\section{SPIELENDE MASCHINEN. MATERIALISIERTE MEDIALITÄT}

Ein Spiel ohne Spieler zu denken, mag als recht abstraktes Gleichnis für eine Selbstbewegung oder eine originäre Produktivität genommen wer-

\section{Ebd., S. 222. \\ Ebd., S. 223.}

29 Ebd. Der Begriff der Erscheinung wird dabei von Fink nicht subjektorientiert oder existential gedacht, sondern auf das »Spiel der Welt« bezogen. Dieses »Weltspiel« wird nicht auf den Maßstab menschlicher Wahrnehmung bezogen, sondern allgemeiner auf Prozesse der Individuierung. Es wird nicht etwas dem Menschen zur Erscheinung gebracht, sondern die »Dinge ins Erscheinen « zu bringen gehört zum Spiel der Welt in dem Sinne wie auch das Weltall "spielt«. Finks Überlegungen gehören also eng zu seiner Deutung des Spiels als kosmisches Gleichnis. In diesem ist das Spiel vom Menschen abgelöst, gleichzeitig ist für Fink der Mensch ein Spieler, genau weil er 'weltlich $<$ ist (ebd., S. 221). Diese Oszillation der Spielbegriffe zwischen anthropologischer und kosmologischer Perspektive bildet das Grundmotiv im Schlussteil von Finks Spiel als Weltsymbol (S. 218-24). 
den, als Gedankenbild. Springt man jedoch vom >Begriff< in die 'Geschichte', wird in spielenden Maschinen auf unterschiedliche Weise ein Spiel ohne Spieler medien- und technikgeschichtlich manifest. Innerhalb der Computerkultur treten Spiel und Technik in konkrete enge Beziehung. Spielende Maschinen, und solche bringt die technologische und medienkulturelle Geschichte des Computers vielfach hervor, lassen sich als Herausforderung für den Spielbegriff verstehen. Wendet man die medienmaterielle Realität spielender Maschinen theoretisch um, dann lässt sich die These aufstellen, dass in der spielenden Maschine ein Verhältnis von Spiel und Technik zur Darstellung kommt, in dem menschliche Subjektivität und Bewegungsform der Natur, anthropologischer und kosmologischer Spielbegriff mediengeschichtlich in Vermischung gebracht sind. Zwei mediengeschichtlich relevante Beispiele hierfür sind Computerschach und Game of Life (kurz: >Life «). Sie rücken jeweils auf unterschiedliche Weise ein Spielen in den Blick, das sich von den spielenden menschlichen Subjekten ablöst und als selbsttätige Bewegung auf Maschinen abläuft. Technik steht hier zumindest teilweise auf Seiten eines nicht-personalen oder automatischen Spielens. Für die Frage der Aufteilung des Spielbegriffs sind diese medialen Spiele deshalb von besonderem Interesse, weil sie weder einem kulturalistischen noch einem formalistischen Spielbegriff einseitig zuzuordnen sind, sondern zwischen beiden eigentümlich changieren.

\section{COMPUTERSCHACH}

Computerschach und 'Life $<$ markieren unterschiedliche Stationen in der Mediengeschichte des Computers: Schach steht in den 1950ern modellhaft für ganz grundsätzliche Fragen der Programmierbarkeit der neuen Rechenmaschinen, und avanciert später zum Lieblingsspiel der Artificial-Intelligence-Forschung. 'Lifer dagegen wird Ende der 1960er Jahre erfunden und gehört einer anderen Phase der Computergeschichte an, in der Programmierung langsam beginnt, in der Populärkultur Resonanz zu finden.

Die formale Nähe zwischen Spielen und Computerprogrammen wurde schon häufig beschrieben. Es ist bekannt, dass prägende Figuren des frühen Computerdiskurses wie Claude Shannon, Alan Turing, Stanislaw Ulam, Allen Newell, Herbert Simon und John von Neumann intensiv Regelspiele untersuchten, um daraus Gewinn für die Entwick- 
lungsmöglichkeiten des Computers und seiner Programmierung zu ziehen. Computerschach ist das prominenteste Beispiel für diese mediengeschichtliche Affinität zwischen Spiel und Software. Claude Shannons berühmter Text Programming a Computer for Playing Chess etablierte in den 1950ern Schachprogrammierung als zentrale Problemstellung und Denkmodell für die Konzeption von Software. ${ }^{30}$ Ausgehend vom Schachspiel formulierte er die Grundlagen für ein neues Paradigma von Programmierung, verstanden als algorithmische Formulierung von >intelligenten< Lösungsstrategien und Erkundung neuer Programmiertechniken, im Unterschied zum etablierten Paradigma des Rechenautomaten als Maschine für effektives >Number-Crushing ${ }{ }^{31}$

Mediengeschichtlich ist Shannons Arbeit zu Schach mit dem Kontext der Diskussionen zu Maschinen-Intelligenz verbunden, die in den 1950er Jahren intensiv geführt wurden, insbesondere im Schnittfeld von Mathematik, Techniktheorie, Computerforschung und Philosophie. Im Artificial-Intelligence Diskurs werden Spiele schließlich vermittelt über Schach zum Prüfstein für die Differenzbestimmung von >Rechnen` und

30 Claude E. Shannon, »Programming a Computer for Playing Chess (1950) «, in Claude Elwood Shannon. Collected Papers, hg. v. N.J.A. Sloane u. Aaron D. Wyner (New York: IEEE, 1993), S. 637-56.

31 Vgl. weiterführend zur Rolle von Schach im frühen Computerdiskurs Serjoscha Wiemer, "Strategiespiele und das Medium-Werden des Computers. Computerschach als Faktor der Rekonzeptionalisierung des Computers als programmierbare Maschine zwischen 1945 und 1960", in Diskurse des Strategischen Spiels. Medialität, Gouvernementalität, Topografie, hg. v. Stefan Böhme u.a. (Münster: LIT, 2014), S. 83-112. Die Sonderrolle von Schach für die AI-Forschung stellt Ensmenger dar. Nathan Ensmenger, »Is Chess the Drosophila of Artificial Intelligence? A Social History of an Algorithm «, Social Studies of Science, 42 (2011), S. 5-30. Die Herausforderungen, die Shannon anhand von Computerschach aufzeigt (Symbolverarbeitung, Problemlösen, Such-Algorithmen, Entscheidungsfindung), sind derart anspruchsvoll, dass sie unmittelbar von höheren Programmiersprachen profitieren. Solche wurden in den 1950ern erst allmählich entwickelt. Für den Beitrag von Computerschach für die Entwicklung von höheren Programmiersprachen ist konkret in der Arbeit von Newell, Clifford, Shaw und Simon ein Beispiel gegeben. Sie verwendeten in den frühen 1950ern eigene Sprachkonstrukte in der von ihnen entwickelten Information Processing Language für die Programmierung von Schach (Allen Newell, John Clifford Shaw und Herbert Alexander Simon, »Chess-Playing Programs and the Problem of Complexity «, IBM Journal of Research and Development, 2.4 (1958), S. 320-35). IPL gilt als wichtige Inspirationsquelle für die Entwicklung von LISP. 
>Denken`. Über Spiele könnten, so die Leitidee, menschliche Fähigkeiten, die mit Denken, Vernunft, Rationalität und Entscheidungsfähigkeit assoziiert werden, auf Maschinen übertragen werden. Sogar der berühmte Turing-Test, der die (Un)unterscheidbarkeit zwischen Mensch und Computer in einer Spielanordnung (als Frage-Antwort-Spiel) zur Disposition stellt, wurde ursprünglich als Schachpartie entworfen. Die Intelligenz der Maschine sollte sich experimentell darstellen, exakt anhand der Spielweise und der Fähigkeit, sintelligente` Züge zu tätigen. Diese medienanthropologische Aufladung von Computerschach motivierte fortgesetzte Auseinandersetzungen mit der Formalisierbarkeit von Entscheiden, Planen, Bewerten als Rationalisierungen des Denkens; mediengeschichtlich schreibt sie sich fort in der Entwicklung von Programmiersprachen, mit denen Lösungsstrategien der Schachprogrammierung bearbeitet werden.

\section{, LEBENDIGE、 AUTOMATEN}

Game of Life ist ebenfalls Spiel und Darstellungsmedium medientechnischer Innovationsbemühungen. Anders als bei Schach ist das Generalthema jedoch nicht die Übertragung von `Intelligenz auf Maschinen, sondern die Nachbildung von Qualitäten des `Lebendigen`. John von Neumann hatte in den 1940er Jahren ein Konzept selbstreproduzierender Automaten entworfen, als er über die Grenzen der technischen Weiterentwicklung von Maschinen nachdachte. ${ }^{32}$ Evolutionäre Anpassungen, zufällige Mutationen und die Fähigkeit von Lebewesen zur Selbstreproduktion verstand er als Schlüssel für Prozesse der Komplexitätssteigerung, die Lebewesen von Maschinen grundsätzlich unterschieden. Mit selbstreproduzierenden Automaten sollte diese Differenz zwischen Lebendigem und künstlichen Maschinen reduziert werden: Selbstreproduzierende Automaten sollten Eigenschaften des Lebendigen nachbilden, um evolutionäre Komplexitätssteigerungen auf Maschinen zu übertragen.

Von Neumann hatte in den 1940ern verschiedene Varianten solcher Automaten erdacht. ${ }^{33}$ Als der Mathematiker John Horton Conway Ende

\footnotetext{
32 Seine Überlegungen wurden durch A. Burks zusammengetragen und posthum veröffentlicht. John von Neumann, Theory of Self-Reproducing Automata, hg. v. Arthur W. Burks (Urbana: University of Illinois Press, 1966).

33 Einen frühen Überblick über die kinetischen und zellulären Automaten gibt 
der 1960er Jahre zu zellulären Automaten arbeitet, knüpft er an diese Forschungen an und sucht nach einer möglichst einfachen Konfiguration, durch die Selbstreproduktion und Komplexitätssteigerung mathematisch zu modellieren wären. Und er findet die Lösung in Form eines Spiels, das durch wenige Regeln definiert werden kann und das als Game of Life berühmt wird. ${ }^{34}$ Bereits auf der Ebene der Benennung der Spielelemente als 'Zellen`, die in Abhängigkeit zu ihren Nachbarn >überleben`, ssterben` oder ‘geboren werden`, evoziert das Spiel die Frage der `Nachbildung von Lebendigem im technischen Spiel. Tatsächlich wurde und wird >Life< vielfach zur Modellierung >natürlicher` und >lebensähnlicher Prozesse verwendet. Im Diskurs der computerbasierten Forschungen zu künstlichem Leben wird es sogar als Beispiel für die These herangezogen, " auch nichtorganische Dinge als lebendige anzuerkennen « ${ }^{3}$

Ein Teil der Attraktivität des Spiels besteht in seiner visuellen Qualität, der Eigenschaft, unvorhergesehene Musterbildungen hervorzubringen, die immer wieder als visuell faszinierend beschrieben und exemplarisch zur Darstellung von Komplexität und Emergenzprozessen verwendet werden. >Life< macht anschaulich, wie aus einfachen Regeln komplexe und unvorhergesehene Formen entstehen können.

Die Soziologin Sherry Turkle hat ihre erste Begegnung mit dem Spiel beschrieben und ihre Erzählung vermittelt etwas von der Faszination, die von >Life ausgehen kann:

Meine erste Bekanntschaft mit dem Spiel machte ich an einem kleinen Bildschirm, der zufällig frei war. Objekte bewegten sich aufeinander zu und strebten wieder auseinander, Formen bildeten sich, verschwanden und tauchten erneut auf. Ich entsinne mich noch, daß ich an Feuer und Wasser denken mußte. Der französische Philosoph Gaston Bachelard hat einmal geschrieben, daß die Beobachtung von Feuer, wie die Betrachtung von strömendem Wasser, etwas sei, das Menschen aller Kulturen und

Shannon. Claude E. Shannon, "Computers and Automata ", Proceedings of the IRE, 41 (1953), S. 1234-41.

34 Nach seiner Veröffentlichung in einer Kolumne für Unterhaltungsmathematik und Puzzlespiele, die von Martin Gardner in Scientific American verantwortet wurde, erlangte das Spiel rasch eine anhaltende Popularität in akademischen wie außerakademischen Kreisen. Es gilt als das bekannteste Beispiel so genannter zellulärer Automaten (CA) und trug dazu bei, die Beschäftigung mit CA innerhalb der Mathematik als eigenständige Forschungsrichtung zu etablieren.

35 Steven Levy, KL - Künstliches Leben aus dem Computer (München: Droemer Knaur, 1993), S. 16. 
aller Zeiten fasziniert habe. Beide Phänomene zeichneten sich durch Wiederholung und Identität, Überraschung und Ordnung aus. Feuer und Wasser rufen Erinnerungen an die ewigen Muster des Lebens wach; und ein Computerbildschirm tat mittlerweile das gleiche. Im Jahre 1977 stand ich allein vor dem Bildschirm, beobachtete das Game of Life und fühlte mich wie ein kleines Mädchen am Ufer eines Ozeans. ${ }^{36}$

Bemerkenswert an Turkles Beschreibung ist, wie fraglos das Spiel hier als Darstellung von >Lebendigkeit< und als bewegte Formen von Feuer oder Wasser wahrgenommen wird. Ihre Schilderung lässt an Gadamers Konzept von Spiel als Bewegungsform der Natur denken. Was Turkle allerdings beschreibt, sind keine in der Natur beobachtbaren Musterbildungen, sondern Qualitäten einer medientechnisch realisierten Spielkonfiguration, Qualitäten tanzenden Lichts, Visualisierungen algorithmischer Abläufe, sichtbar auf einem Computerbildschirm. Diese Überschneidungen von Turkles mit Gadamers Spielbegriff sind ästhetisch begründet; was als Spiel der Natur im Sinne Gadamers anschaulich wird, ist qua Spiel medial vermittelt als Anschauung einer spielenden Maschine.

\section{MEDIENANTHROPOLOGISCHER AUSBLICK}

Die knapp dargestellten Computer-Spiele, Schach und >Life`, wurden gewählt, um die mediengeschichtliche Konkretisierung der Verbindung von Spiel und Technik zu exemplifizieren. Beide Spiele oszillieren auf besondere Weise zwischen ludischen und epistemischen Praxen, sind offen für spielerische und wissenschaftliche Herangehensweisen und verweisen auf eine Nähe oder gar Überschneidung und Durchdringung von Spiel und Experiment, Spiel und Simulation, Spiel und Wissensproduktion.

Deutlich ist in jedem Fall, dass spielende Maschinen die gängigen Aufteilungen des Spielbegriffs in Frage stellen können. Sie fordern dazu heraus, ein Spiel ohne Subjekt zu denken, ein Spiel das sich weitgehend selbsttätig entfaltet. Ein solches Spiel setzt den Menschen nicht als autonomes und zentrales Subjekt für seinen Ablauf. Stattdessen werden medienanthropologische Fragestellungen anderer Art aufgerufen, weil

36 Sherry Turkle, Leben im Netz: Identität in Zeiten des Internet (Reinbek bei Hamburg: Rowohlt, 1998), S. 247-48. 
durch mediale Spiele ein Wissen vom Menschen produziert wird (die denkende Maschine als Schachprogramm bei Turing) oder weil die Abgrenzungen, Übergänge und Übersetzungen zwischen Maschinen und dem Lebendigen in der Form medialer Spiele zur Anschauung gebracht werden (die lebensähnlichen Musterbildungen von >Life $<$ ). ${ }^{37}$ Nimmt man die medienanthropologische Dimension dieser Spiele ernst, ihre medienund technikgeschichtliche sowie epistemologische Position, ${ }^{38}$ dann lässt sich verstehen, wie Spiele zwischen Mensch und Technik vermitteln, gerade indem sie anthropozentrische Spielbegriffe in Frage stellen.

In dieser Richtung ließe sich auch die Medialität des ComputerSpiels versuchsweise fassen: Die Medialität dieser Spiele drückt sich darin aus, wie sie etwas zur Anschauung und zur Darstellung bringt, das selbst veränderlich sich zeigt, nämlich das mediengeschichtlich sich wandelnde Verhältnis von Mensch und Technik. ${ }^{39}$ Das Spiel wäre dann als Teil einer reflexiven Bewegung zu verstehen, die dem Menschen die Möglichkeit eröffnet, im Spiel und als Spiel sein Verhältnis zur Technik und seine Stellung zur Welt zu befragen.

Offen bleibt, wie die Analogie zwischen Spiel und Naturbewegung, die bei >Life< deutlich wurde, angesichts formalistischer, ontologischer und kosmologischer Spielbegriffe zu bewerten ist. Es stellt sich die Frage, ob in einem Spiel wie >Life r vielleicht ein technischer Begriff von Natur

37 Selbstverständlich sind es nicht allein die Spiele, von denen aus Großbegriffe wie >Mensch oder >Natur in Frage gestellt werden. Die Spiele sind Knotenpunkte in technisch-diskursiven Gefügen. Sie verknüpfen und vermitteln zwischen unterschiedlichen Technologien und Diskursen, als deren materielle Artikulationen sie zugleich verstanden werden können.

38 Vgl. weiterführend zu medienanthropologischen Fragestellungen unter Berücksichtigung von Spiel und Technik: Astrid Deuber-Mankowsky, »Mediale Anthropologie, Spiel und Anthropozentrismuskritik «, Zeitschrift für Medien- und Kulturforschung (2013), S. 133-48.

39 Wie in diesem Zusammenhang das Verhältnis von Spiel und Technik vorgestellt werden kann, ist von entscheidender Bedeutung und verdiente eine eigene Untersuchung. Einen Ausgangspunkt dafür können Walter Benjamins Überlegungen zu Spiel und Zweiter Technik darstellen, wie sie jüngst durch Astrid Deuber-Mankowsky in die Diskussion gebracht worden sind, insbesondere wegen ihrer medientheoretischen und medienanthropologischen Relevanz. Benjamins 'Zweite Technik « verbindet - über das Spiel -, wie Deuber-Mankowsky zeigt, die Frage nach dem Verhältnis von Natur und Menschheit mit einer möglichen »Kritik des Kapitalismus und der Kritik der Wertschöpfung aus der menschlichen Arbeit «, vgl. weiterführend Deuber-Mankowsky, »Spiel und zweite Technik«, S. 60-62. 
sich artikuliert, in dem Natur und Technik tendenziell ununterscheidbar werden, oder ob in der Verbindung von Spiel und Technik vielleicht sogar ein ’kosmologisches< Fundament von Technik zur Anschauung kommen kann. Wenn man mit Fink davon ausgeht, dass im Spiel ein originäres produktives Weltverhältnis sich ausdrückt und Form gewinnt, dass es sowohl als anthropologisches als auch als kosmologisches Schlüsselphänomen verstanden werden kann, dann wäre vielleicht auch das Spiel der Technik nach beiden Seiten hin offen.

Eine medienanthropologische Reflexion des Spielbegriffs steht vor der Aufgabe, die historisch sich verändernde Art und Weise zu beschreiben, wie Spiele als Medien der Selbstreflexion des Menschen fungieren und wie es um ihren Anteil an der Hervorbringung und Stabilisierung des Wissens bestellt ist, das den Menschen konstituiert. Wenn es möglich ist, die gängigen Polarisierungen des Spielbegriffs in einer solchen medienanthropologischen Perspektive zu überschreiten, würde damit vielleicht auch das Spiel als >kosmologischer Schlüssel und als Bewegungsform der Natur mit aufgenommen werden, um jenseits eines anthropozentrischen Spielbegriffs dafür aufmerksam zu werden, welches Potenzial dem Spiel als Vermittlung zwischen Mensch, Technik und Natur mediengeschichtlich zugewachsen ist. 
Serjoscha Wiemer, »Niemandes Spiel?: Zur Aufteilung des Spielbegriffs oder: Die Schwierigkeit, die spielende Maschine zu denken «, in Denkweisen des Spiels: Medienphilosophische Annäherungen, hg. v. Astrid Deuber-Mankowsky und Reinhold Görling, Cultural Inquiry, 10 (Wien: Turia + Kant, 2017), S. 155-70 <https: //doi.org/10.37050/ci-10_09>

\section{QUELLENANGABEN}

Caillois, Roger, Méduse \& Cie (Berlin: Brinkmann \& Bose, 2007)

Die Spiele und die Menschen. Maske und Rausch (Frankfurt a.M., Berlin, Wien: Ullstein, 1982)

Corbineau-Hoffmann, A., »Spiel «, in Historisches Wörterbuch der Philosophie, hg. v. Joachim Ritter und Karlfried Gründer (Basel: Schwabe \& Co., 1998), IX (1998), S. 1383-90

Derrida, Jacques, $\gg$ Die différance $\ll$, in ders., Randgänge der Philosophie (Wien: Passagen-Verlag, 1988), S. 31-52

Deuber-Mankowsky, Astrid, »Mediale Anthropologie, Spiel und Anthropozentrismuskritik «, Zeitschrift für Medien- und Kulturforschung (2013), S. 133-48 <https://doi.org/10.28937/ 1000106386>

— $\gg$ Spiel und zweite Technik. Walter Benjamins Entwurf einer Medienthropologie des Spiels «, in Mediale Anthropologie, hg. v. Christiane Voss u. Lorenz Engell (Paderborn: Fink, 2015), S. 35-62

Ensmenger, Nathan, $\gg$ Is Chess the Drosophila of Artificial Intelligence? A Social History of an Algorithm «, Social Studies of Science, 42 (2011), S. 5-30 <https://doi.org/10.1177/ 0306312711424596>

Gadamer, Hans-Georg, Wahrheit und Methode. Grundzüge einer philosophischen Hermeneutik, 2. durch e. Nachtr. erw. Aufl. (Tübingen: Mohr (Siebeck), 1965 [1960])

Huizinga, Johan, Homo Ludens. Vom Ursprung der Kultur im Spiel (Reinbek bei Hamburg: Rowohlt, 2006)

Koller, Hermann, Die Mimesis in der Antike, Dissertationes Bernenses: Serie 1, Fasc. 5 (Bernae, Bern: Francke, 1954)

Krämer, Sybille, »Form als Vollzug oder: Was gewinnen wir mit Niklas Luhmanns Unterscheidung von Medium und Form? «, Rechtshistorisches Journal, 17 (1998), S. 558-73

Levy, Steven, KL - Künstliches Leben aus dem Computer (München: Droemer Knaur, 1993)

von Neumann, John, Theory of Self-Reproducing Automata, hg. v. Arthur W. Burks (Urbana: University of Illinois Press, 1966)

von Neumann, John und Oskar Morgenstern, Theory of Games and Economic Behavior, hg. v. Ariel Rubinstein und Harold W. Kuhn (Princeton, NJ: Princeton University Press, 2007)

Newell, Allen, John Clifford Shaw und Herbert Alexander Simon, »Chess-Playing Programs and the Problem of Complexity«, IBM Journal of Research and Development, 2.4 (1958), S. 320-35 <https://doi.org/10.1147/rd.24.0320>

Shannon, Claude E., »Computers and Automata «, Proceedings of the IRE, 41 (1953), S. 1234-41 $<$ https://doi.org/10.1109/JRPROC.1953.274273>

— Programming a Computer for Playing Chess $(1950) \ll$, in Claude Elwood Shannon. Collected Papers, hg. v. N.J.A. Sloane u. Aaron D. Wyner (New York: IEEE, 1993), S. 637-56

Turkle, Sherry, Leben im Netz: Identität in Zeiten des Internet (Reinbek bei Hamburg: Rowohlt, 1998) 